\title{
Maternal serum homocysteine and uterine artery Doppler as predictors of preeclampsia and poor placentation
}

\section{Ahmed Mohamed Maged $^{1}$}

Published online: 30 November 2017

○) Springer-Verlag GmbH Germany, part of Springer Nature 2017

\section{Dear Editor,}

First of all, I would like to thank Dr. Pek and Dr. Beyazi for their interest in our research and their valuable comments and observations.

It is a known fact that the level of homocysteine is affected by both serum B12 and folic acid. However, the deficiency of these factors can be easily assessed through their obvious effects on RBC formation. In the exclusion criteria, we excluded women with chronic diseases and the most important of them was anemia of any cause. All our pregnant women were investigated through complete blood picture and count (being part of the routine investigations offered according to Kasr Alainy medical school recommendation) and all women with anemia of any type were excluded.

Of course, we totally agree with Dr. Bek and Dr. Beyazi in their concerns, we think that serum assessment of B12 and folic acid load our patients with excess costs and should be restricted to those with suspected deficiency as those with macrocytic anemia.

Thank you again for your time and effort.

\section{Compliance with ethical standards}

Conflict of interest Professor Ahmed Maged confirm no conflict of interest.
Ahmed Mohamed Maged

prof.ahmedmaged@gmail.com

1135 King Faisal Street, Haram, Giza 12151, Egypt 